\title{
What makes a human? Conscious human beings are hyperobjects that interact via physical and mental representations in an ecosystem based on fundamental story-flow interactions.
}

Walter Lerchner (ORCID: 0000-0003-0842-4409), Bethesda, MD 20814, lerchner@gmail.com

Summary: What is a human being? Some might answer this question by referring to a biological body, growing from genetic information passed on through generations. Others refer to a mind, developed from infancy to adulthood, expressing itself self-aware and intelligently. Few will argue that a human being could exist without one or the other, but many disagree on their relative contribution. Does the conscious mind emerge solely from a single physical body? Is the developing body shaped purely by biological

predetermination? I propose that the formation of individual human beings is subject to an environment that envelops both, the physical and mental realms. This environment is here referred to as story-verse of humanity. It is an ecosystem that emerged from biological activity but grew and evolved into an interactive space that includes temporal interactions, such as created by nervous system activity. The emerging storyverse gives rise to persistent hyperobjects, including individual human beings, whose stories perpetuate themselves via physical and mental representations. The story-verse is a real physics realm that includes the four fundamental interactions described by particle physics, but additionally requires higher-order fundamental forces that facilitate interactions between the physical and mental realm.

\section{Contents}

Introduction

The Ship of Theseus

How do physical objects manifest, persist, and interact?

How do mental objects manifest, persist and interact?

How do we represent mental ideas in the physical realm?

The central nervous system perceives physical objects in seven dimensions

Human beings are emotional, rational, and empathetic

What the evolving stories of a screwdriver and Santa Claus have in common

Fundamental Interactions building the human story-verse

\section{Introduction}

The question, "what is man?" has been subject to a heated debate spanning religious, philosophical, psychological and biological texts throughout the recorded history of mankind (Carroll et al. 2008; Darwin 1871; Descartes and Hall 1972; Kandel 2018). Depending on the viewpoint of the person asking, the answer has been focusing on the body, the conscious mind, or a spiritual self that is beyond either of these. One of 
the more famous quotes is: "cogito ergo sum", "I think, therefore I am" by Descartes (Descartes 1960), which has been interpreted as a separation of mind from body and led some modern thinkers, as well as Sci-Fi authors, to consider the possibility of "uploading" the mind, and thus the human self, into purely electronic existence (Kurzweil 2010). Others, such as Antonio Damasio, countered with "I feel, therefore I am" (Damasio 1994), arguing that the human self is found in the physical responses of the body, and that our mind is merely reacting to and representing physical processes. Spiritual texts in eastern and western philosophies argue that our existence is found neither in the body nor the mind, but rather in a soul, or a spirit, independent of body and thought (Eckhart and Walshe 2009; Jalāl al-Dīn and Wilson 1976). While there is much disagreement between these different roads of argumentation, many would agree that a full description of conscious, self-aware human beings, is currently beyond the understanding of physics and biology. I will present a case that, indeed the essence of what makes us human cannot be solely prescribed to the body or the thinking mind but is nonetheless accessible by a scientific, physics-based, description.

\section{The Ship of Theseus}

At the core of any being, whether physical or imaginary, must be the question of "self". What gives an "entity" its unique individual nature? A thought experiment famous for pondering this question is the Ship of Theseus (Plato and Reeve 1998). Is a ship, that had all its parts replaced over time, still the same ship? I suggest that the question should be different: Why do the parts of this ship get replaced in a way that allows it to persist? The answer to what the actual Ship of Theseus is, lies in its story. The "self" that keeps

reinforcing its existence is the purpose of the story, i.e., the (mutual) benefit that it brings to its environment. The objective appearance of the Ship of Theseus is expressed through the understanding of the story, i.e., how it interacts with its environment. No combination of physical objects, nor any combination of mental ideas alone could form a physical Ship of Theseus, without the story bringing them together into just such a persistent form. I argue here that the environment in which a Ship of Theseus would exist is the human story-verse, an ecosystem of stories that allows it to form and persist by perpetuating physical and mental processes that sustain its existence. In the story-verse, the Ship of Theseus has a single continuous identity, but may have many representations in the physical realm as material ships, and in the mental realm as varying narratives. Thoughts that should arise when contemplating the above are: What is a story 
exactly, how could it be described using physics, and how does it exert causal agency onto the physical and mental realms? These are questions that we are going to try to answer below.

\title{
How do physical objects manifest, persist, and interact?
}

Before we can understand the causal reality of stories, we first need to reevaluate how we describe the formation and interaction of physical, particle-based, objects within an ecosystem created by the fourdimensional spacetime environment. And we need to understand how physical objects can be faithfully represented in an ecosystem within the mental realm. This requires us to dive into some physics and geometry. I have described the principles of such an ecosystem framework elsewhere (Lerchner 2020), but the basics should suffice here. Ecosystems are built on interactive flows between participating objects. Interactions within an ecosystems act self-reinforcing, or autocatalytic, to shape the environment such that participation creates mutual benefit for the individual and the whole, i.e., makes each more likely to persist than without each other (Ulanowicz 1995). Once a sustainable ecosystem forms, it becomes an identity that goes through developmental stages from growth and differentiation to maturity and stability (Ulanowicz 1997). Providing selection pressure to its (internal) subsystems, it also becomes an interactive, persistent, object in its own (external) environment (Fig. 1).

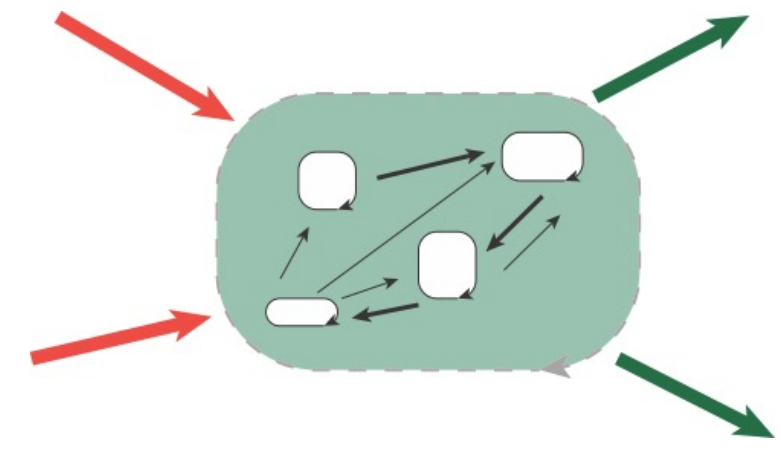

\author{
Figure 1. Every object is an ecosystem with an internal \\ and external environment. When interactions between \\ objects become mutually beneficial, they create an internal \\ self-reinforcing flow environment that evolves these \\ interactions via natural selection of subsystems towards a \\ stable state of maximal fitness (Ulanowicz 1997). Towards its \\ external environment it presents a unified adaptive object \\ identity (Varela 1974). Every subsystem also has an internal \\ ecosystem. Arrows indicate examples for stable interactions.
}

Applying this viewpoint to the spacetime environment of the physical universe, we can describe any type of particle-based object, such as atoms, hurricanes, viruses and organisms as interactive entities nested within the physical realm. Particle physics tells us that all massive objects in the universe arise from four fundamental interactions that act between elementary particles: the strong force, the weak force, electromagnetism and gravity. When physical spacetime is viewed as an ecosystem, these same interactions are considered fundamental flow forces constraining a four-dimensional flow surface, 
comparable to the surface of a flowing river. Instead of vortexes and ripples forming and being carried downstream on a river surface, physical objects form on the spacetime surface and are carried along the flow of time. And instead of forming from interactions of air- and water molecules on a river surface, physical objects form from interactions of particles on a spacetime surface. The behavior of any physical object or process can be described by fundamental interactions via the Schrödinger equation, but this gets tedious for complex systems, as it must be done at the level of elementary particles. The ecosystem viewpoint instead argues that the same principles that apply to the interactions of elementary particles, apply to the interaction of objects of any size and shape. In the ecosystem framework, fundamental interactions describe dimensional interactions on a flow surface, with those interacting between elementary particles being only a special case (elementary interactions). Fundamental interactions are more generally explained as: object to object interactions (i1), mass flow gradient interactions (i2), conformational pattern interactions (i3), and spatiotemporal interactions (i4). As illustrated in Table 1, these correspond directly with the strong force (i1), weak force (i2), electromagnetism (i3) and gravity (i4) with (in) indicating interactive dimensionality. If the viewpoint is correct, then a type of Schrödinger equation should exist to model the behavior of systems at any level of complexity.

\begin{tabular}{|l|l|l|l|l|l|}
\hline dimensions & $\begin{array}{l}\text { fundamental } \\
\text { interaction }\end{array}$ & $\begin{array}{l}\text { elementary } \\
\text { interaction }\end{array}$ & $\begin{array}{l}\text { internal } \\
\text { force }\end{array}$ & objective force & $\begin{array}{l}\text { causal } \\
\text { manifestation }\end{array}$ \\
\hline i1 & point to point & strong force & kinetic & repulsion & movement \\
\hline i2 & gradient to gradient & weak force & surface & transformation & mass flow \\
\hline i3 & pattern to pattern & electromagnetism & conformation & information & $\begin{array}{l}\text { pattern } \\
\text { replication }\end{array}$ \\
\hline i4 & behavior to behavior & gravity & adaptation & attraction & identity \\
\hline
\end{tabular}

Table 1. Fundamental flow interactions of physical objects. Flow forces dimensionally constrain environmental flows as a result of object identities. Elementary interactions are the result of elementary particles. Fundamental interactions are defined by the dimensionality of interactions (i) and are expressed by any physical object with a self-reinforcing identity.

The next step is to understand how, from such fundamental interactions, all the diverse types of physical objects form. Not unlike conventional physics, the ecosystem framework argues that every system and process within spacetime can be described by these fundamental flow forces. There is a natural hierarchy of four types of emerging objects that are distinguished by their agency of flow dimensionality used to assert selection pressure onto their subsystems. Material objects, like atoms, molecules, planets, and solar systems constrain the acceleration of subsystems via point-to-point interactions (i1). To achieve stable 
acceleration within the system, they create selection pressure on subsystems to evolve subsystems towards a base state of relative rest (si1). Thermodynamic objects, like hurricanes and chemical systems, constrain the feedback loops of subsystems via gradient (mass flow) interactions (i2). These systems develop subsystems towards a state of equilibrium (si2). Genetic objects, like virus strains, constrain replication of subsystems (i3) and evolve subsystems towards a state of homeostasis (si3). Biological objects (organisms), like bacteria colonies and multicellular species, constrain the spatiotemporal behavior of subsystems (i4) and develop subsystems, like cells or organs, towards an evolutionary stable state (si4). Stable objects express a consistent appearance towards their external environment via recurring (nonrandom) interactive flows, and a state of dynamic rest (balance of forces) towards their internal environment. Any external or internal disruption of this state will result in forces by the object that drive its evolution towards regaining a stable state (Ulanowicz 1997). It must be emphasized, that the dynamically stable states discussed here are not only specific to a type of system but are defining the type of system. Another way of defining objects according to the same ontology is by type of attractor. Material objects use acceleration (i1) to attract, well, other material objects. Thermodynamic objects attract energy (mass-flow of objects), via gradients (i2). Genetic objects use their conformations (i3) to attract replicators (information), and organisms use behavior (i4) to attract history (learning) and other identities (e.g., symbiotes) that are of benefit to their evolution. Table 2 summarizes types of physical objects and expression of their flow forces.

\begin{tabular}{|l|l|l|l|l|l|}
\hline dimensions & system type & constraint & stable state & $\begin{array}{l}\text { environmental } \\
\text { disruption }\end{array}$ & causal response \\
\hline si1 & material & acceleration & relativ rest & kinetic impact & movement \\
\hline si2 & theromdynamic & energy conversion & equilibrium & energy exposure & gradient change \\
\hline si3 & genetic & reproduction & homestasis & $\begin{array}{l}\text { conformation } \\
\text { disruption }\end{array}$ & replication change \\
\hline si4 & organism & behavior & $\begin{array}{l}\text { evolutionary } \\
\text { stable state }\end{array}$ & $\begin{array}{l}\text { identity } \\
\text { disruption }\end{array}$ & identity change \\
\hline
\end{tabular}

Table 2. Types of physical objects and expression of their flow forces. Objective identities dimensionally constrain environmental flows via mutual interactions of their subsystems. Higher-dimensional constraints result in more dynamically complex stable states (si).

The ecosystem framework offers an ontology of particle-based systems that is not restricted to particle interactions but is applicable to any interactive entity based on physical spacetime. For example, many interactive system theories describe organisms as "complex", "chaotic" and "far from equilibrium" (Capra and Luisi 2014; Juarrero 1999; Ulanowicz 2009). This is a false narrative, because "equilibrium" 
is not the correct state to describe an objective presence of an organism. An organism is stable when its cells and organs are in an evolutionary stable state (si4) in which the equilibrium (si2) and homeostasis (si3) of its subsystems are dynamically constrained by forces that are neither "complex", nor impossible to model. We might as well describe a gas-filled box, as particles in a state "far from relative rest" and the movement of the gas molecules as complex and chaotic, even though the system itself can be described by a few simple vector forces constraining the dynamic of molecules. Another example of a false narrative is the description of an atom or a proton as not dynamic because there is no "energy exchange". A proton appears objectively stable, but it is "far from equilibrium". Its stable state is the result of an ecosystem dynamically sustained by interacting quarks. $99 \%$ of proton mass does not originate from the mass of the quarks but instead stems from kinetic energy and virtual particles that constantly go through cycles of birth and death (Cho 2008). This is even more obvious for the neutron, which has an average life-expectancy of 15 minutes, when outside the stabilizing environment of the atomic nucleus (Moskowitz 2014).

The most important insight is that all types of objects have real, self-contained identities, including agency over subsystems and a defined representable external appearance. Another important insight is that objects adapt to their environment by changing the selection pressure environment of their subsystems. Finally, we can now apply the same principles to the formation and interaction of mental objects.

\section{How do mental objects manifest, persist and interact?}

We know from physics that the constituents of material objects are elementary particles. What are the constituents of mental objects and how do they interact? Physics tells us that particles are spatial excitations that persist and evolve over time (Carroll 2019). Fundamental interactions between particles that lead to the formation of physical objects require multiple dimensions to interact via kinetics (i1), gradients (i2), patterns (i3), and behaviors (i4) (Table 1). To represent such interactions in the brain, the mental realm too requires multiple interactive dimensions. The brain also must be able to create representations of physical objects from sensory information that arrives temporally disjointed and is processed on different timescales (Wallace and Stevenson 2014). Buonomano goes as far as to call the brain a time machine (Buonomano 2017). The ecosystem framework agrees, but the system that achieves such a feat cannot be 
described by particle interactions alone (e.g., biochemistry) but rather by an additional interactive ecosystem that forms on top of the biological brain. The scientific explanation for mental, or imaginary, objects (concepts and ideas) is that they emerge from neural activity in the central nervous system of individual human beings (Kandel 2018). If we accept this notion, we need to have a closer look of what neural activity actually is. When applying the ecosystem framework, the constituents of neural activity are action potentials, temporal excitations (instances) that persist and evolve in physical spacetime. The interactive flow surface for action potentials is spatially constrained by the network of neurons and synapses, but interactions occur via evolving temporal instances. How can objects emerge from instances? The temporally constrained flows that create the mental ecosystem are fundamental interactions based on propagating action potentials (Table 3): Spikes (incidences) traveling along axons and synapses and interacting with each other, creating a flow of incidences (i1). When multiple spikes sustainably interact, they form a coincidence, evolving their subsystems towards the representation of a present moment in spacetime (si1). Durations (i2) manifest via spike trains (mass-flow of instances) that can vary in length, allowing formation of gradients and feedback loops within a circuit, evolving subsystems towards a state of attention (si2). Analogous to particle-based objects, they act as a gradient (mass-flow) attractors of neural activity. Frequency (i3) manifests as replication of spike train patterns across brain regions, allowing formation of coherence/resonance of activity between brain regions, resulting in reproducible and representable information, that evolves subsystems towards a state of recognition (si3). Narrations (i4) manifest in adaptable spike trains that adaptively change over reproductions, resulting in recurring spike trains that include spatial and temporal identity formations, memories, evolving towards a stable state of identification (si4), allowing spatiotemporal representation of objects and events.

\begin{tabular}{|l|l|l|l|l|l|}
\hline flow dimensions & $\begin{array}{l}\text { fundamental } \\
\text { interaction }\end{array}$ & expression & $\begin{array}{l}\text { object } \\
\text { dimensions }\end{array}$ & mental object & stable state \\
\hline i1 & instances & spike & si1 & coincidence & present \\
\hline i2 & duration & spike train & si2 & circuits & attention \\
\hline i3 & frequency & oscillation & $\mathbf{s i 3}$ & coherence & recognition \\
\hline i4 & narrations & consolidation & $\mathbf{s i 4}$ & memory & Identification \\
\hline
\end{tabular}

Table 3. Fundamental mental flows and corresponding mental objects. Interactions (i) are based on temporally constrained spatial flows. Stable objects constrain subsystems via self-reinforcing dimensional flows (si). 
Fundamental interactions of the mental realm create a representational frame (Kant et al. 1955) able to give rise to the same interactivity possible in the physical realm. Natural laws governing interactions are determined by the biological nervous system and will be different from those in the physical realm. However, the mutual survival of biological species and mental objects creates selection pressure for the interactivity in the mental realm to closely represent interactivity in the physical realm.

\section{How do we represent mental ideas in the physical realm?}

In the above, we established that the physical realm is built within an ecosystem that is constrained in three spatial dimensions and one temporal flow dimension. The mental realm, meanwhile, is built within an ecosystem that is constrained in three temporal dimensions and one spatiotemporal flow dimension. Both realms share the time-flow dimension. Coincidences, durations, frequencies and even narrations appear as sequential occurrences, when viewed from the perspective of spacetime, but mental objects and their interactivity space, form dimensionally orthogonal to spacetime. Below, we will introduce the story-verse as an overlying ecosystem enveloping both, physical and mental interactivity of human beings. Before we get there, we need to address one more question: How can objects of physical and mental ecosystems interact and build representations in the other realm? Human beings appear to do this naturally. We perceive interactive concepts of physical objects in our mind via sensory organs, and we use muscle movements to create technological and artistic representations of mental ideas in the physical world. Imagine the forging of a sword as a representation of a mental idea. Neurons will use temporal modulations of physical muscles to guide heating and cooling processes that force the molecules in the metal to adapt to a desired shape (Hrisoulas 1991); processes that would be extremely unlikely to occur without a mind. To achieve these physically improbably occurrences, we use temporally constrained mental objects (ideas) and our spatially constrained physical body (muscles) to modulate the behavior (i4) of metal molecules via coincidence (i1), duration (i2), frequency (i3) and adaptive narration (i4). In short, we are adding three additional temporal dimensional constraints onto the naturally dissipative behavior of the metal to force its evolution into the desired shape. The resulting object, the sword, will still be a four-dimensional object in the physical world but its creation required a seven-dimensional process. 


\section{The central nervous system perceives physical objects in seven dimensions}

According to the ecosystem framework, mental representations of physical objects form via modulation of the mental narration flow (i4). Physical forces emerging from material objects impact our senses kinetically (mechanically), as gradients (e.g., temperature, sound volume), patterns (taste, smell, light) or behavior (adaptive sequences). These flows spatially modulate neural activity and thus the environment of preexisting mental objects. Like the forging of a sword, physical senses shape mental objects until they correspond to physical counterparts. Kant points out (Kant et al. 1955), and so does Object-OrientedOntology (Harman 2018), that mental objects are created from phenomena of physical objects, i.e., from their external appearances. The conclusion is that mental representations can never fully correspond to the reality of physical objects and may not even have a physical counterpart. This is also correct within the interpretation of the ecosystem framework, but as discussed with the example of a sword, this does not make mental ideas unreal. On the contrary, mental objects in conjunction with the biological nervous system can add layers of complexity, allowing interactivity of objects and representations that go beyond the particle interactivity of physical spacetime.

For example, when perceiving a sword, we represent its physical appearance in our mind: its shape, material, weight, etc. However, this is not all we perceive. A sword also creates emotional, rational, and even empathetic connotations in our mind that are dependent on its outward appearance, as well as circumstances under which it was forged and previously used. This means, that although the sword is a four-dimensional object made from particle interactions, our mind perceives it as a higher-dimensional object with additional interactive dimensional characteristics. Not only does our mind perceive it this way but perceiving it can have real causal effects on our interactions with other beings and objects in the physical realm. If the representational frame of our mind is four-dimensional, how can we represent higher interactive dimensions of objects? The human central nervous system is not only made from neural activity, nor is it only a biological system, but it provides an ecosystem to both, biological and mental processes. The brain responds to neural activity and vice versa, creating an interactive feedback system in which up to seven dimensions of a physical object can be represented. It must be pointed out here that we are not talking about metaphysical or purely mathematical dimensions, but interactions within three physical space 
dimensions plus four temporal constraints expressed by coincidence, duration, frequency, and adaptive narration. When perceiving natural physical objects via our senses, there are not just spatial interactions involved. For example, if we hear sound, we not only perceive the kinetic impact, but we also perceive a varying level of volume, which is a temporal gradient of air pressure in addition to its dynamic spatial distribution. This makes sound waves, even when ignoring frequency, a five-dimensional flow vector (i5). Bats use such flows for detailed mapping of their environment, something impossible without temporal processing. Similarly, when we use vision to perceive light distribution, we perceive the spatial distribution of light rays emanating from an object, but we also perceive the frequency distribution, information that according to our framework requires three temporal constraints, making light, i.e., the spatial patterning of photon impact, a six-dimensionally constrained flow (i6). Finally, to uniquely identify an object, we combine sequential changes of sensory flows (narration) with the location of an object in space, resulting in a sevendimensionally constrained flow (i7). Thus, the interactive dimensionality of perceived is not a virtual tool, but these are real physics flows with spatial and temporal constraints. The nervous system and the neural activity interacting with in, just allows us to perceive these flows.

\section{Human beings are emotional, rational, and empathetic}

In the above section, I argued that the nervous system itself can represent up to seven-dimensional interactions using the laws of physics. I also mentioned that human can imbibe physical objects with emotional, rational, and empathetic characteristics, both when creating and when perceiving them. In the following sections, I dive deeper on how these aspects fit together, and how every-day interactions of human beings occur in an even higher-dimensional interactive space, the human story-based ecosystem. The first clue of such a realm is found in physical objects that require groups of human beings: higher-order social systems. According to the ecosystem framework, such systems are direct results of physical behavior flows that are modulated by mental flows and expressed by muscle movement. Such modulated interactive flows are expressed by individuals as: On/Off sequences of behaviors (i4), e.g., habits and dance routines; gradations of behaviors (i5), e.g., fast or slow to communicate emotional emphasis or as an expression of motivation towards a goal; rule-based behaviors that can be imitated (i6), e.g., instructions for solving a problem or constructing a building; dialectic behaviors that adapt to the environment of the 
interaction (i7), e.g., an interaction that leads to a realization of a new mental or social object, such as the process of forming and sustaining a family. Social entities based on habitual laws evolve their subsystems towards a stable state of obedience (si4); entities based on ideology evolve subsystems towards a state of certainty (si5); entities based on science evolve subsystems towards a state of knowledge (si6); and entities based on empathy/mutual benevolence evolve subsystems towards a state of understanding (si7). By social entities, I am not referring to groups of humans, but to social objects forming from their interactions. As we will see below, a full description of human interactions, requires even higher-dimensional flows. Table 4 summarizes social flows, objects and their corresponding objective expressions.

\begin{tabular}{|l|l|l|l|l|l|}
\hline $\begin{array}{l}\text { flow } \\
\text { dimensions }\end{array}$ & behavior flow & expression & $\begin{array}{l}\text { object } \\
\text { dimensions }\end{array}$ & object type & stable state \\
\hline i4 & sequence (on/off) & habits & si4 & laws & obediance \\
\hline i5 & motivation (graded) & goals & si5 & ideology & certainty \\
\hline i6 & rule-based (frequency) & imitation & si6 & science & knowledge \\
\hline i7 & dialectic (adaptive) & realization & si7 & empathy & understanding \\
\hline
\end{tabular}

Table 4. Emergence of social objects from temporally modulated behavior flows. Higher-order interactive flows (i) are expressed by any social object. For social objects, like for lower-order physical objects, higher-dimensional constraints result in more dynamically complex stable states (si).

\section{What the evolving stories of a screwdriver and Santa Claus have in common}

Finally, we return to questions on the nature of stories. Up to now all objects that we considered where either part of the physical realm or the mental realm, even though their formation might have been a result of their interactive modulation, e.g., a work of abstract art from a mental idea, a fear-inducing perception as the result of a defense mechanism by an organism, or a city or an organization as the result of human interaction. If the mental realm and the physical realm are complementary ecosystems, in which "medium" or via which "ecosystem" are interactions between these two realms conveyed? I propose an ecosystem of stories that includes all physical and mental interactions discussed, and at least three higher-dimensional constraints. How is this possible and why don't we measure such "forces" in our physics experiments? The answer to the latter question is: "we do, but we don't account for them as such" (Lerchner 2020). As to the question how this is possible, let's look at the actual physical and intellectual power of story objects. 
The first example for a story-based object may sound harmless enough. The evolving story of the screwdriver. We are not talking about a single specific screwdriver. We are referring to a story born from the interaction of a screw and mental ideas, as to the purpose of improving the turning of a screw, which then led to a back and forth of evolutionary iterations of physical screws, screwdrivers and corresponding mental ideas concerned with them. The manifestation of the first physical screwdriver likely had a specific time and place, maybe sometime in the $15^{\text {th }}$ century (Rybczynski 2000). However, subsequently the screwdriver object became an ecosystem that expanded in both, mental and physical realms, with temporally and spatially constrained subsystems that can interact with each other. The screwdriver ecosystem is a large hyperobject (Morton 2013) that continuous to give rise to many physical screwdrivers, as well as varying concepts, ideas for improvement and emotions of excitement and frustration. It gave rise to work and riches for generations of human beings. Like other technologies, its evolution most certainly followed an S-Curve of growth, differentiation, maturation, and stability (Arthur 2009; Ulanowicz 1997). Its representations, physical screwdrivers, graphic depictions of screwdrivers, or associated voice utterances, can uniquely identify it in the conscious mind of most human beings, without them needing to be in physical contact.

The second example may express even more powerful influence onto physical and mental realms: The story of Santa Claus. There might have been a singular moment of space and time, when this story took shape, maybe when an individual observed the actions of a physical person. Or, like for the origin of a river, different story streams combining to form the unique object of Santa Claus. There is no question; however, that the story grew over time and manifested in many variations around the globe. In all its versions, it is uniquely recognizable and compels humans to create physical and mental representations that propagate and sustain it. In return, the story of Santa Claus causes sustenance of beneficial economic and emotional processes in many individual humans. So next time when you tell a child that Santa Claus is real, you can do so, knowing that it is true.

Both these examples of story objects are part of an ecosystem that we intuitively call humanity. This ecosystem does not just include physical human beings. It includes objects such as clothing, houses, engines and farm animals that extent our physical presence. It includes electronic information, in which 
spatially travelling electronic instances extend the mental realm. It also includes tradition, knowledge and mutual understanding that cannot be propagated by any physical or mental process alone but require interaction of many such processes and beings. I call this ecosystem the story-based system of humanity. What are the fundamental interactions on which such an ecosystem is built upon?

\section{Fundamental Interactions building the human story-verse}

It may make sense to first understand how a single human individual expresses agency onto the physical and mental world. The biological body uses DNA encoded templates to grow from a single cell into a behaving organism via adaptive recurring changes in cells and organs. Thus, the body itself is an object with (some) agency over all four dimensions of spacetime. This is the case not only for human bodies but also for bacteria colonies, plants and fungi. However, the human body also contains a nervous system giving rise to a mental ecosystem of up to seven-dimensional objects (understanding - si7). Both systems (neural-activity based objects and the biological body) share the time-flow axis of spacetime but are otherwise complementary parts of a single interactive system. Therefore, a human being can use mind and body to express up to ten-dimensional interactions (i10) that result in up to ten-dimensional stable systems (si10). Maybe even more relevant, these dimensionally constrained objects are not limited to the physical or the mental realm but can be sustained by any combination of flows. In the story-based system of humanity, stories can be represented in the physical realm and/or perceived as mental ideas. But their propagating identities, exist within and across human beings, taking advantage of interactivity in the physical and mental realms. Nonetheless, story objects are real physics-based objects that emerge from self-reinforcing interactions and have a unique objective presence. From a physics point of view, they are unusual because they are neither spatially, nor temporally localized. Rather, their objective expressions and resulting forces are often distributed widely across the physical and mental realms.

According to the ecosystem framework, interactions between object are defined by dimensionality. Depending on the environment that they occur in, they will manifest with varying characteristics. If we propose up to ten-dimensional interactive forces between objects in the human story-verse, there should be correlates of such interactive forces in physics. Indeed, I suggest that there are. Current viewpoints in 
physics assume that what we call "natural laws" are unchanging environmental properties from which physical manifestations emerge. When viewed within the ecosystem framework, many of such laws should arise directly from geometric characteristics of dimensionally constrained flow forces. In Table 5, I propose an ontology of up to 10 dimensional fundamental forces that express themselves in corresponding physics and human story-based manifestations. If assumptions in this table are validated, the framework should allow generalized descriptions of objects across fields as diverse as physics, psychology, and sociology.

\begin{tabular}{|c|c|c|c|c|c|}
\hline $\begin{array}{l}\text { interactive } \\
\text { dimensions }\end{array}$ & fundamental interaction & expression & physics manifestion & story-flow interaction & expression \\
\hline 1 & particles & matter & strong force & acceleration & motion \\
\hline 2 & gradients & energy & weak force & transformation & radiation \\
\hline 3 & conformations & information & electromagnetic force & reproduction & construction \\
\hline 4 & spacetime identity & history & gravity & adaptation & propagation \\
\hline 5 & spacetime gradient & time & higgs field & desires & reward \\
\hline 6 & spacetime patterns & light & quantum coherence & meaning & imitation \\
\hline 7 & objective identity & cohesion & quantum decoherence & language & rules \\
\hline 8 & objective transformation & believe & probability & ideas & ideology \\
\hline 9 & objective reproducibility & knowledge & fractionality & rationality & science \\
\hline 10 & objective reality & understanding & unity & empathy & benevolence \\
\hline
\end{tabular}

Table 5. Fundamental interactions and their proposed manifestation in physical and story phenomena. Interactions or flows manifest dimensionally, therefore higher-order flows include properties of lower-order flows.

We mentioned two actual story objects in the screwdriver story and the Santa Claus story that can be considered vast hyperobjects, spanning the globe. However, every single human being is also such a hyperobject, with agency within both the mental and physical realms. Travel via cars, boats and planes can expand the physical expression of a human being across the globe and into the solar system. Electronic communication, e.g., via cell phone and/or the internet, can expand the mental expression of a single individual across the human sphere of influence. The ecosystem framework discussed here, has many more implication on physics and psychological viewpoints, some which have been addressed previously (Lerchner 2020). For us humans, it might help us to better understand our own powers, responsibilities and potentials. As a story-based object, humanity has already conquered the planet and started to expand into 
the solar system, but in terms of a ten-dimensional being, based on mutually benevolent understanding, capable of empathy for any other object in the story-verse, we are still at the very beginning of our

evolution.

\section{References}

Arthur, W. Brian. 2009. The nature of technology : what it is and how it evolves (Free Press: New York).

Buonomano, Dean. 2017. Your brain is a time machine : the neuroscience and physics of time (W. W. Norton \& Company: New York).

Capra, Fritjof, and P. L. Luisi. 2014. The systems view of life : a unifying vision (Cambridge University Press: Cambridge).

Carroll, Robert, Robert P. Carroll, Stephen Prickett, and Inc NetLibrary. 2008. The Bible : Authorized King James Version. Psalm 8:4-6 (Oxford University Press: Oxford ; New York).

Carroll, Sean M. 2019. Something deeply hidden : quantum worlds and the emergence of spacetime (Dutton, an imprint of Penguin Random House: Boston, Massachusetts).

Cho, Adrian. 2008. 'At Long Last, Physicists Calculate the Proton's Mass', Science.

Damasio, Antonio R. 1994. Descartes' error : emotion, reason, and the human brain (Putnam: New York).

Darwin, Charles. 1871. The descent of man, and selection in relation to sex (D. Appleton and company: New York,).

Descartes, René. 1960. Discourse on method, and Meditations (Liberal Arts Press: New York,).

Descartes, René, and Thomas S. Hall. 1972. Treatise of man (Harvard University Press: Cambridge).

Eckhart, and Maurice O'C Walshe. 2009. The complete mystical works of Meister Eckhart (Crossroad Pub. Co.: New York).

Harman, Graham. 2018. Object-oriented ontology : a new theory of everything (Pelican Books: London).

Hrisoulas, Jim. 1991. The master bladesmith : advanced studies in steel (Paladin Press: Boulder, Colo.).

Jalāl al-Dīn, Rūmī, and C. E. Wilson. 1976. The Mašnavī (Indus Publications: Karachi).

Juarrero, Alicia. 1999. Dynamics in action : intentional behavior as a complex system (MIT Press: Cambridge, Mass.).

Kandel, Eric R. 2018. The disordered mind : what unusual brains tell us about ourselves (Farrar, Straus and Giroux: New York).

Kant, Immanuel, J. M. D. Meiklejohn, Thomas Kingsmill Abbott, James Creed Meredith, Immanuel Kant, Immanuel Kant, and Immanuel Kant. 1955. The critique of pure reason (Encyclopædia Britannica: Chicago,).

Kurzweil, Ray. 2010. The Singularity is Near (Gerald Duckworth \& Co: London).

Lerchner, Walter. 2020. 'Human agency over mind and matter: A physics framework for describing the objective reality of material-, neural activity- and story-based entities. ', OSF Preprints.

Morton, Timothy. 2013. Hyperobjects : philosophy and ecology after the end of the world (University of Minnesota Press: Minneapolis). Moskowitz, Clara. 2014. 'Neutron death mystery has physicists stymied', Nature.

Plato, and C. D. C. Reeve. 1998. Cratylus (Hackett Pub. Co.: Indianapolis).

Rybczynski, Witold. 2000. One good turn : a natural history of the screwdriver and the screw (Scribner: New York).

Ulanowicz, R. E. 1995. 'Utricularias Secret - the Advantage of Positive Feedback in Oligotrophic Environments', Ecological Modelling, 79: 49-57.

Ulanowicz, Robert E. 1997. Ecology, the ascendent perspective (Columbia University Press: New York).

--- . 2009. A third window : natural life beyond Newton and Darwin (Templeton Foundation Press: West Conshohocken, Pa.).

Varela, F.G., Maturana, H.R., Uribe R. 1974. 'Autopoiesis: The organization of living systems, its characterization and a model', Biosystems, 5: 187-96.

Wallace, M. T., and R. A. Stevenson. 2014. 'The construct of the multisensory temporal binding window and its dysregulation in developmental disabilities', Neuropsychologia, 64: 105-23. 